\title{
Analytical Modeling of Hard-Coating Cantilever Composite Plate considering the Material Nonlinearity of Hard Coating
}

\author{
Wei Sun, ${ }^{1}$ Ying Liu, ${ }^{2}$ and Guangyu Du${ }^{1}$ \\ ${ }^{1}$ School of Mechanical Engineering \& Automation, Northeastern University, Shenyang 110819, China \\ ${ }^{2}$ Institute of Vibration, Shock and Noise, State Key Laboratory of Mechanical System and Vibration, \\ Shanghai Jiao Tong University, Shanghai 200240, China
}

Correspondence should be addressed to Wei Sun; ddsunwei@126.com

Received 5 May 2015; Revised 25 June 2015; Accepted 27 August 2015

Academic Editor: Anna M. Gil-Lafuente

Copyright (C) 2015 Wei Sun et al. This is an open access article distributed under the Creative Commons Attribution License, which permits unrestricted use, distribution, and reproduction in any medium, provided the original work is properly cited.

\begin{abstract}
Due to the material nonlinearity of hard coating, the coated structure produces the nonlinear dynamical behaviors of variable stiffness and damping, which make the modeling of hard-coating composite structure become a challenging task. In this study, the polynomial was adopted to characterize this material nonlinearity and an analytical modeling method was developed for the hard-coating composite plate. Firstly, to relate the hard-coating material parameters obtained by test and the analytical model, the expression of equivalent strain of composite plate was derived. Then, the analytical model of hard-coating composite plate was created by energy method considering the material nonlinearity of hard coating. Next, using the Newton-Raphson method to solve the vibration response and resonant frequencies of composite plate and a specific calculation procedure was also proposed. Finally, a cantilever plate coated with $\mathrm{MgO}+\mathrm{Al}_{2} \mathrm{O}_{3}$ hard coating was chosen as study case; the vibration response and resonant frequencies of composite plate were calculated using the proposed method. The calculation results were compared with the experiment and general linear calculation, and the correctness of the created model was verified. The study shows the proposed method can still maintain an acceptable precision when the material nonlinearity of hard coating is stronger.
\end{abstract}

\section{Introduction}

In recent years, vibration control using hard coating which is prepared by the metal, ceramic, or their mixture is receiving a reasonable amount of attention. Compared with traditional surface treatments, such as viscoelastic dampers, the biggest technical advantage is that the hard coating can maintain the consistent damping characteristics in the high temperature or high corrosion environment. Thus, this vibration control technology is expected to apply in the power equipment, such as the turbine engine. From the characterizing test studies $[1,2]$ about the coated composite structure, some interesting phenomenon has been found; for example, the resonant frequency decreased with the increase of excitation amplitude and the force response of frequency were not symmetric about the resonant frequency. But for the uncoated structure, the above-mentioned phenomenon did not happen, which proves that the hard coating makes the composite structure have the nonlinear vibration phenomenon. Further studies show that this nonlinearity comes from the strain dependent characteristic of coating material, that is, the storage modulus (or Young's modulus) and loss modulus of hard coating change with the strain response amplitude of structure, and belongs to the material nonlinear behavior.

To effectively implement the hard-coating damping technology, the reliable analysis model, which can reproduce the nonlinear dynamic phenomenon observed in the experiment, need be created. However, to the authors' opinions, there is much bigger gap away from this goal. As far as the current research status is concerned, the modeling about hard-coating composite structure is mainly based on the micro material science. For example, Tassini et al. [3] created a phenomenological model to characterize the nonlinear mesoscopic elastic behaviors of hard-coating 


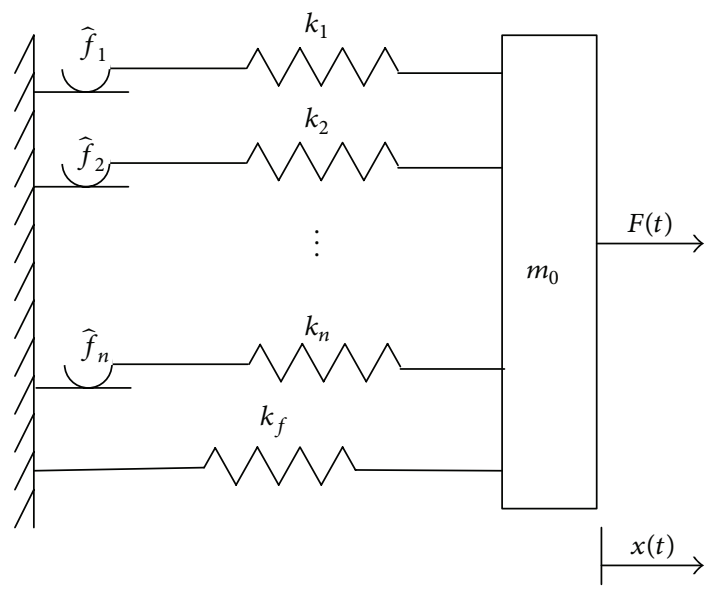

FIGURE 1: Iwan model.

material. Torvik [4] developed a slip damping model to describe the idealized microstructure characteristic of hard coatings. Al-Rub and Palazotto [5] proposed a representative volume element-based micromechanical model to observe the nonlinear damping in plasma sprayed hard ceramic coatings. All the above studies show that the internal friction in the coating creates the energy dissipation of composite structure, being very important to understand the nonlinear dynamics mechanism or vibration reduction mechanism of hard coating. However, for the hard-coating damping design, it is not realistic that entirely depending on these micro material models and developing the dynamics model based on the macro vibration science are necessary. Furthermore, the challenge of developing this model comes from how to consider the material nonlinearity of hard coating.

To introduce the material nonlinearity of hard coating into the analysis model, the researchers have proposed several methods. Considering the hard-coating dissipate energy by the internal friction of coating particles, then, some researchers attempt to use the Iwan model (shown in Figure 1) to simulate the strain dependent characteristic of hard coating.

In this model, the storage and loss modulus of hard coating are expressed as spring stiffness, $k_{i}, k_{f}$, and friction forces, $\hat{f}_{i}, i=1,2, \ldots n$, and both of them are the functions of strain amplitude. $F(t)$ is the externally applied force, $m_{0}$ is the mass of the system, and $x(t)$ is the displacement of the mass. Some typical examples show as follows. Green and Patsias [6] applied the Iwan model to describe the response of a coated beam and analyzed the dissipation mechanism of hard coating. Reed [7] did a similar research and used the single-freedom nonlinear adjusted Iwan model to analyze the free response and forced response of hardcoating beam and verified the rationality of relative damping identification method. To effectively use the Iwan model, some key parameters, such as spring stiffness, friction forces, mass, and applied force, should be determined firstly. But it is not easy and the above studies did not show yet how these parameters were selected. Another method is using the stored energy per unit volume, $U\left(\varepsilon_{e}\right)$, and dissipated energy per unit volume, $D\left(\varepsilon_{e}\right)$, to describe the material nonlinearity of hard coating, which is given by

$$
\begin{aligned}
& E_{\mathrm{cR}}\left(\varepsilon_{e}\right)=\frac{2 U\left(\varepsilon_{e}\right)}{\varepsilon_{e}^{2}}, \\
& E_{\mathrm{cI}}\left(\varepsilon_{e}\right)=\frac{D\left(\varepsilon_{e}\right)}{\left(\pi \varepsilon_{e}^{2}\right)},
\end{aligned}
$$

where $E_{\mathrm{cR}}\left(\varepsilon_{e}\right)$ and $E_{\mathrm{cI}}\left(\varepsilon_{e}\right)$ are the storage modulus and the loss modulus of hard coating, respectively, and $\varepsilon_{e}$ is the equivalent strain. The original intention of this expression is to satisfy the need of deducing the identification formula of the material parameters with strain dependent characteristic. For example, Torvik and Hansel $[8,9]$ utilized this expression and obtained the identification formula of material properties of a ceramic coating. The last characterizing idea is applying the polynomial to describe the material parameters of hard coating and this characterizing method is also adopted in this study, which will be introduced in the following section. The benefits of using a polynomial to characterize the material parameters of hard coating are mainly reflected in two aspects. On the one hand, the polynomial can more accurately describe the material parameters of hard coating obtained by experiment. For example, all the discrete point values of hard-coating material parameters gotten by Patsias et al. [10], Reed et al. [11], and Tassini et al. [12] can be described by the polynomial. Furthermore, it is found that high order polynomial should be chosen to fit these discrete point values. On the other hand, the material parameters expressed by polynomial can be easily introduced into the analytical model during the macro vibration analysis. Recently, using the finite element method (FEM) to solve the dynamics of hard-coating composite structure introducing the polynomial expression of hard-coating material parameters has been able to achieve. For example, Filippi and Torvik [13] adopted the linear polynomial to simulate the hard-coating parameters with strain dependent characteristic and analyzed the vibration of compressor blade coated hard coating. However, to the authors' knowledge, the analytical modeling of hard-coating composite structure with the polynomial expression of hard-coating material parameters cannot be found.

On the basis of considering the material nonlinearity of hard coating, the purpose of this study was to develop the analytical modeling method for the hard-coating cantilever thin plate structure. This paper is organized as follows. In Section 2, to relate the material parameters with strain dependent characteristic of hard coating obtained by test and the analytical modeling of hard-coating composite plate, the expression of equivalent strain of composite plate was derived. In Section 3, the analytical model of hard-coating composite plate was created by energy method considering the material nonlinearities of hard coating. In Section 4, Newton-Raphson method was used to solve the vibration response and resonant frequencies of composite plate and a specific calculation procedure was proposed. In Section 5, a cantilever plate coated with $\mathrm{MgO}+\mathrm{Al}_{2} \mathrm{O}_{3}$ hard coating was chosen as study case, and the vibration response and resonant 


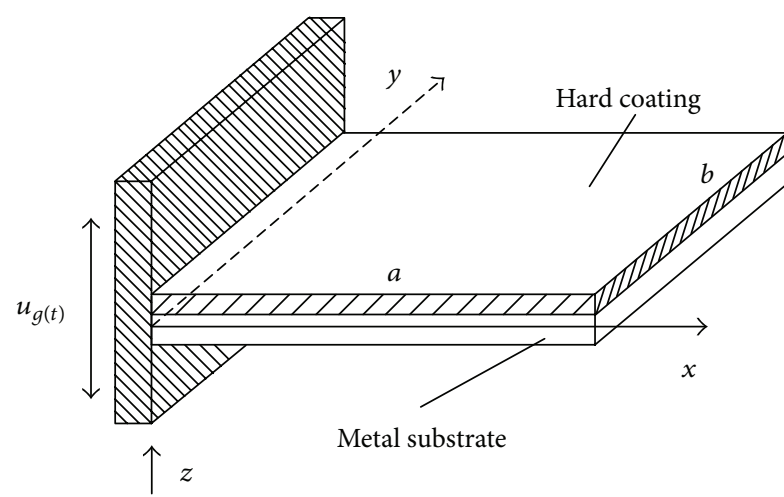

(a) Hard-coating composite plate

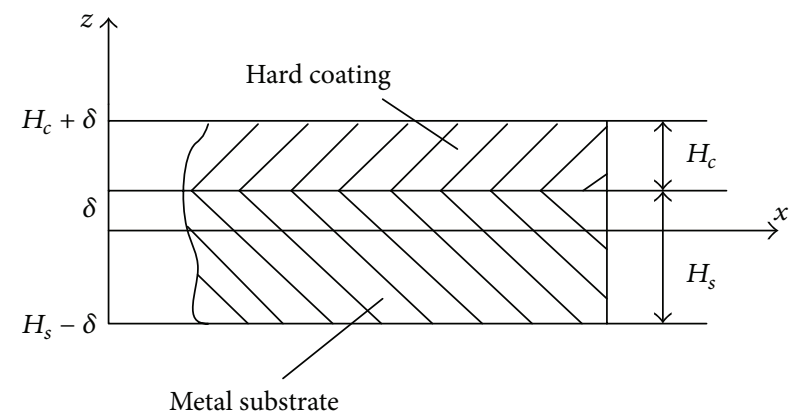

(b) Cross section of composite plate

FIGURE 2: Hard-coating composite plate under base excitation.

frequencies of composite plate were calculated using the proposed analytical model and calculation procedures. The calculation results were compared with the experiment and linear calculation, and the correctness of the created model was verified. Finally, some important conclusions about this study were listed in Section 6.

\section{Derivation of Equivalent Strain for the Hard-Coating Thin Plate Structure}

As above mentioned, during the study of identification of material parameters for the hard coating, the relative curve between the strain amplitude and the storage modulus or loss modulus is determined. Furthermore, these relative curves can be fitted by polynomial, and then the material parameters with strain dependent characteristic are described effectively. Both the finite element modeling and analytical modeling are needed to introduce these mechanical parameters with polynomial expression correctly. For the beam structure, only a unidirectional strain can characterize the dynamics of beam, and corresponding to this unidirectional strain, the material parameters expressed by polynomial can be introduced easily. However, for the plate structure in this study, even the classical plate theory is adopted, and at least three kinds of strain still need to be adopted to describe the dynamics of plate. Then, how to relate the material parameters obtained by test and the analytical model? In this section, the equivalent strain of hard-coating composite plate was chosen to relate the measured material parameters expressed by polynomial and the analytical model. In the following, a brief introduction about the polynomial expression of hardcoating material was given firstly, and then the equivalent strain of hard-coating thin plate structure was derived.

The elastic modulus of hard coating is expressed as complex modulus $E_{c}^{*}$ and the following formula can be used to describe strain dependent characteristic:

$$
E_{c}^{*}=E_{\mathrm{cR}}\left(\varepsilon_{e}\right)+i E_{\mathrm{cI}}\left(\varepsilon_{e}\right),
$$

where ${ }^{*}$ refers to complex value.
When the complex modulus of hard coating is expressed by polynomial, the storage and loss modulus in (2) change as

$$
\begin{aligned}
& E_{\mathrm{cR}}\left(\varepsilon_{e}\right)=E_{\mathrm{cR} 0}+\varepsilon_{e} E_{\mathrm{cR} 1}+\varepsilon_{e}^{2} E_{\mathrm{cR} 2}+\varepsilon_{e}^{3} E_{\mathrm{cR} 3}+\cdots, \\
& E_{\mathrm{cI}}\left(\varepsilon_{e}\right)=E_{\mathrm{cI} 0}+\varepsilon_{e} E_{\mathrm{cl} 1}+\varepsilon_{e}^{2} E_{\mathrm{c} 22}+\varepsilon_{e}^{3} E_{\mathrm{c} 13}+\cdots,
\end{aligned}
$$

where $E_{\mathrm{CR} 0}$ and $E_{\mathrm{CI} 0}$ are the storage and loss modulus without considering the strain dependent characteristic of hard coating, and $E_{\mathrm{cR} j}, E_{\mathrm{cI} j}(j=1,2,3, \ldots)$ are the specific coefficients of strain dependent storage modulus and loss modulus. Therefore, integrating (2)-(3), the complex modulus of hard coating can be also expressed as

$$
E_{c}^{*}=E_{c 0}^{*}+E_{c 1}^{*} \varepsilon_{e}+E_{c 2}^{*} \varepsilon_{e}^{2}+E_{c 3}^{*} \varepsilon_{e}^{3}+\cdots,
$$

where

$$
\begin{aligned}
& E_{c 0}^{*}=E_{\mathrm{cR} 0}+i E_{\mathrm{cI} 0}, \\
& E_{c j}^{*}=E_{\mathrm{cR} j}+i E_{\mathrm{cI} j}, \quad j=1,2,3, \ldots .
\end{aligned}
$$

In (4), the material parameters of hard coating can be divided two parts: one is $E_{c 0}^{*}$, and the other is $E_{c 1}^{*} \varepsilon_{e}+E_{c 2}^{*} \varepsilon_{e}^{2}+$ $E_{c 3}^{*} \varepsilon_{e}^{3}+\cdots$. For $E_{c 0}^{*}$, it does not include the strain dependent characteristic, so it can be named as linear part of hardcoating materials. On the contrary, $E_{c 1}^{*} \varepsilon_{e}+E_{c 2}^{*} \varepsilon_{e}^{2}+E_{c 3}^{*} \varepsilon_{e}^{3}+\cdots$ can be called nonlinear part of hard-coating materials.

The schematic diagram of hard-coating composite plate is shown in Figure 2, and the thin plate is in cantilever status and is excited by base. The reason of choosing this boundary condition is that the base excitation was adopted to test the vibration characteristics in many studies $[1,2]$. For the most coating structures, of course including the hard-coating composite plate structure of this study, all of them belong to thin shell structure; that is, the thickness is far less than the length and width or other geometry dimensions. So, herein the hard-coating thin plate can be analyzed according to the classic thin plate theory. Only the material nonlinearity of hard coating should be introduced into the analytical model. Since the classic thin plate theory is suitable to deal with this problem, then the movement of any point in the composite 
plate can be described by the transverse displacement (or deflection) of neutral surface.

The $x y$ coordinate plane is set to locate at the neutral surface of composite plate; the length and width of plate are $a$ and $b$, respectively. The deflection in the neutral surface of composite plate is $w(x, y, t)$. The expression of base excitation is $u_{g}(t)=U_{G} e^{i \omega t}$, where $U_{G}$ is the level of base excitation and $\omega$ is the angular frequency of excitation. Figure 2(b) shows a small part of the plate section. Here, $H_{c}$ and $H_{s}$ represent the thickness of the hard coating and the metal substrate, respectively, and $\delta$ is the distance between the interface of coating-substrate and the neutral surface.

According to the classic thin plate theory, the strain of any point in the composite plate can be expressed as

$$
\begin{gathered}
\varepsilon_{x}=-z \frac{\partial^{2} w}{\partial x^{2}} \\
\varepsilon_{y}=-z \frac{\partial^{2} w}{\partial y^{2}} \\
\gamma_{x y}=-2 z \frac{\partial^{2} w}{\partial x \partial y},
\end{gathered}
$$

where $\varepsilon_{x}, \varepsilon_{y}$ are the normal strain of $x$ and $y$ direction, respectively, $\gamma_{x y}$ is the shear strain in the $x y$ plane, and $z$ is the distance between the point of the cross section and neutral surface.

Furthermore, according to the physical equations, the stress of any point in the composite plate can be given:

$$
\begin{aligned}
\sigma_{x} & =\frac{E_{e}}{1-\mu_{e}^{2}}\left(\varepsilon_{x}+\mu_{e} \varepsilon_{y}\right) \\
& =-\frac{E_{e}}{1-\mu_{e}^{2}} z\left(\frac{\partial^{2} w}{\partial x^{2}}+\mu_{e} \frac{\partial^{2} w}{\partial y^{2}}\right), \\
\sigma_{y} & =\frac{E_{e}}{1-\mu_{e}^{2}}\left(\varepsilon_{y}+\mu_{e} \varepsilon_{x}\right) \\
& =-\frac{E_{e}}{1-\mu_{e}^{2}} z\left(\frac{\partial^{2} w}{\partial y^{2}}+\mu_{e} \frac{\partial^{2} w}{\partial x^{2}}\right), \\
\tau_{x y} & =G_{e} \gamma_{x y}=-2 G_{e} z \frac{\partial^{2} w}{\partial x \partial y},
\end{aligned}
$$

where $\sigma_{x}, \sigma_{y}$ are the normal stress of $x$ and $y$ direction, respectively, $\tau_{x y}$ is the shear strain in the $x y$ plane, $E_{e}, \mu_{e}$ are equivalent Young's modulus and Poisson's ratio, respectively (it should be noted that Poisson's ratio in this work is thought as independent with the strain amplitude), and $G_{e}$ is the equivalent shear modulus and the relation with equivalent Young's modulus and Poisson's ratio can be expressed as

$$
G_{e}=\frac{E_{e}}{2\left(1+\mu_{e}\right)}
$$

Here, the equivalent strain of hard-coating composite plate was determined according to the principle of equal strain energy density; the expression is

$$
\sigma_{x} \varepsilon_{x}+\sigma_{y} \varepsilon_{y}+\tau_{x y} \gamma_{x y}=\sigma_{e} \varepsilon_{e}
$$

where $\sigma_{e}$ is the equivalent stress and satisfies

$$
\sigma_{e}=E_{e} \varepsilon_{e}
$$

Equations (6), (7a), (7b), and (7c), and (10) can be substituted into (9) and then yield

$$
\begin{aligned}
& \frac{E_{e} z^{2}}{1-\mu_{e}^{2}}\left[\left(\frac{\partial^{2} w}{\partial x^{2}}\right)^{2}+2 \mu_{e}\left(\frac{\partial^{2} w}{\partial x^{2}}\right)\left(\frac{\partial^{2} w}{\partial y^{2}}\right)+\left(\frac{\partial^{2} w}{\partial y^{2}}\right)^{2}\right. \\
& \left.+2\left(1-\mu_{e}\right)\left(\frac{\partial^{2} w}{\partial x \partial y}\right)^{2}\right]=E_{e} \varepsilon_{e}^{2} .
\end{aligned}
$$

Thus, the equation of solution of equivalent strain can be obtained and shown as

$$
\begin{aligned}
\varepsilon_{e}^{2} & =\frac{z^{2}}{1-\mu_{e}^{2}}\left[\left(\frac{\partial^{2} w}{\partial x^{2}}\right)^{2}+2 \mu_{e}\left(\frac{\partial^{2} w}{\partial x^{2}}\right)\left(\frac{\partial^{2} w}{\partial y^{2}}\right)\right. \\
& \left.+\left(\frac{\partial^{2} w}{\partial y^{2}}\right)^{2}+2\left(1-\mu_{e}\right)\left(\frac{\partial^{2} w}{\partial x \partial y}\right)^{2}\right] .
\end{aligned}
$$

It should be noted that because of introducing the complex response in the subsequent analytical modeling of composite plate, the deflection $w(x, y, t)$ of composite plate is also a complex. For simplification of expression, only material parameters were marked as complex in this study. The equivalent strain amplitude should be a real, so the solution formula is changed as

$$
\varepsilon_{e}=\sqrt{\frac{z^{2}}{1-\mu_{e}^{2}}\left|\left(\frac{\partial^{2} w}{\partial x^{2}}\right)^{2}+2 \mu_{e}\left(\frac{\partial^{2} w}{\partial x^{2}}\right)\left(\frac{\partial^{2} w}{\partial y^{2}}\right)+\left(\frac{\partial^{2} w}{\partial y^{2}}\right)^{2}+2\left(1-\mu_{e}\right)\left(\frac{\partial^{2} w}{\partial x \partial y}\right)^{2}\right|}
$$

where the operator $|\cdot|$ refers to solving the modulus of complex values.
The calculation in (13) is very complex, which will reduce the computational efficiency obviously for the subsequent 
iterations, so a relatively simple expression of equivalent strain amplitude is further given:

$$
\begin{aligned}
\varepsilon_{e} & \approx \varepsilon_{e, \max } \\
& =\frac{z}{\sqrt{1-\mu_{e}^{2}}}\left(\left|\frac{\partial^{2} w}{\partial x^{2}}\right|+\left|\frac{\partial^{2} w}{\partial y^{2}}\right|+\sqrt{2\left(1-\mu_{e}\right)}\left|\frac{\partial^{2} w}{\partial x \partial y}\right|\right) .
\end{aligned}
$$

The final expression is corresponding to the maximum value of equivalent strain amplitude in (13) and practice has shown that this simplification does not introduce big error but improves the computational efficiency greatly.

\section{Analytical Modeling of Hard-Coating Composite Plate}

In the following, the energy method was adopted to create the analytical model of the hard-coating composite plate (shown in Figure 2) under the base excitation. Usually, the loss factor of hard coating is higher than the common metals, but it is much less than the viscoelastic materials, so the damping of hard coating belongs to medium or low levels. Then, the damping contribution of both hard coating and metal substrate was considered in this study. Here, the complex modulus model was used to describe both hard coating and metal substrate. The complex modulus expression of hard coating can be seen from (2) to (5), and the modulus of metal substrate is expressed as

$$
E_{s}^{*}=E_{\mathrm{sR}}+i E_{\mathrm{sI}}
$$

where $E_{\mathrm{sR}}, E_{\mathrm{sI}}$ are the storage modulus and the loss modulus of metal substrate, and both of them were set as constant in this study.

The distance $\delta$ between the interface of coating-substrate and the neutral surface can be determined as [14]

$$
\delta=\frac{E_{\mathrm{sR}} H_{s}^{2}-E_{\mathrm{cR} 0} H_{c}^{2}}{2\left(E_{\mathrm{sR}} H_{s}+E_{\mathrm{cR} 0} H_{c}\right)} .
$$

Due to undertaking the base excitation, the displacement $d(x, y, t)$ of any point in the composite plate can be expressed as

$$
d(x, y, t)=u_{g}(t)+w(x, y, t) .
$$

It can be noted from (17) that if the deflection $w(x, y, t)$ of any point is known, then the displacement $d(x, y, t)$ can be determined.

The energy in the hard-coating thin plate system should include strain energy, kinetic energy, and damping dissipation energy, and these energies will be solved below. According to the classic thin plate theory, the strain energy of hard-coating plate system can be given as

$$
U=\frac{1}{2} \iiint_{V}\left(\sigma_{x} \varepsilon_{x}+\sigma_{y} \varepsilon_{y}+\tau_{x y} \gamma_{x y}\right) \mathrm{d} V=U^{l}+U^{\mathrm{nl}}
$$

where $V$ refers to the volume of composite plate and $U^{l}$ and $U^{\mathrm{nl}}$ are named as the linear and nonlinear strain energy, respectively, which are corresponding to the linear and nonlinear part of hard-coating material parameters.

Introducing the constitutive model of substrate and linear part of hard coating, respectively, and integrating along $z$ axis, the solution formula of linear strain energy can be yielded:

$$
\begin{array}{r}
U^{l}=\frac{1}{2} \iint_{A}\left\{D_{1}\left[\left(\frac{\partial^{2} w}{\partial x^{2}}\right)^{2}+\left(\frac{\partial^{2} w}{\partial y^{2}}\right)^{2}\right]\right. \\
\left.\quad+2 D_{2} \frac{\partial^{2} w}{\partial x^{2}} \frac{\partial^{2} w}{\partial y^{2}}+D_{3}\left(\frac{\partial^{2} w}{\partial x \partial y}\right)^{2}\right\} \mathrm{d} A,
\end{array}
$$

where $A$ refers to the area of composite plate, and the variables, such as $D_{1}, D_{2}$ and $D_{3}$, can be expressed as

$$
\begin{aligned}
& D_{1}=\frac{E_{s}^{*} N_{1}}{1-\mu_{s}^{2}}+\frac{E_{c 0}^{*} N_{2}}{1-\mu_{c}^{2}}, \\
& D_{2}=\frac{E_{s}^{*} N_{1} \mu_{s}}{1-\mu_{s}^{2}}+\frac{E_{c 0}^{*} N_{2} \mu_{c}}{1-\mu_{c}^{2}}, \\
& D_{3}=4 G_{s}^{*} N_{1}+4 G_{c 0}^{*} N_{2}, \\
& N_{1}=\frac{1}{3}\left[\delta^{3}+\left(H_{s}-\delta\right)^{3}\right], \\
& N_{2}=\frac{1}{3}\left[\left(H_{c}+\delta\right)^{3}-\delta^{3}\right],
\end{aligned}
$$

where $\mu_{s}, \mu_{c}$ are Poisson's ratios of metal substrate and hard coating. Similarly, the strain dependent characteristics of these Poisson ratios are not considered in the analytical model. $G_{s}^{*}, G_{c 0}^{*}$ are shear modulus of metal substrate and hard coating corresponding to the linear part of hard-coating material.

Furthermore, the nonlinear part of hard-coating material is introduced into the solution of nonlinear strain energy of hard-coating plate. To correspond to the polynomial expression of hard-coating material, the equivalent strain is adopted to calculate the nonlinear strain energy, and the solution formula can be described as

$$
\begin{aligned}
U^{\mathrm{nl}} & =\frac{1}{2} \iiint_{V_{c}}\left(E_{c 1}^{*} \varepsilon_{e}+E_{c 2}^{*} \varepsilon_{e}^{2}+E_{c 3}^{*} \varepsilon_{e}^{3}\right) \varepsilon_{e}^{2} \mathrm{~d} V \\
& =\frac{1}{2} \iint_{A}\left(D_{c 1} f_{\mathrm{eq}} g+D_{c 2} f_{\mathrm{eq}}^{2} g+D_{c 3} f_{\mathrm{eq}}^{3} g\right) \mathrm{d} A,
\end{aligned}
$$


where $V_{c}$ is the volume of hard coating and the expressions of other parameters are shown as follows:

$$
\begin{aligned}
D_{c 1}= & \frac{E_{c 1}^{*}}{4\left(1-\mu_{c}^{2}\right)^{3 / 2}}\left[\left(H_{c}+\delta\right)^{4}-\delta^{4}\right], \\
D_{c 2}= & \frac{E_{c 2}^{*}}{5\left(1-\mu_{c}^{2}\right)^{2}}\left[\left(H_{c}+\delta\right)^{5}-\delta^{5}\right] \\
D_{c 3}= & \frac{E_{c 3}^{*}}{6\left(1-\mu_{c}^{2}\right)^{5 / 2}}\left[\left(H_{c}+\delta\right)^{6}-\delta^{6}\right] \\
f_{\mathrm{eq}} & \sqrt{\operatorname{Re}^{2}\left(\frac{\partial^{2} w}{\partial x^{2}}\right)+\operatorname{Im}^{2}\left(\frac{\partial^{2} w}{\partial x^{2}}\right)} \\
& +\sqrt{\operatorname{Re}^{2}\left(\frac{\partial^{2} w}{\partial y^{2}}\right)+\operatorname{Im}^{2}\left(\frac{\partial^{2} w}{\partial y^{2}}\right)} \\
& +\sqrt{2\left(1-\mu_{c}\right)} \sqrt{\operatorname{Re}^{2}\left(\frac{\partial^{2} w}{\partial x \partial y}\right)+\operatorname{Im}^{2}\left(\frac{\partial^{2} w}{\partial x \partial y}\right)}
\end{aligned}
$$

$g$

$$
\begin{aligned}
= & \left(\frac{\partial^{2} w}{\partial x^{2}}\right)^{2}+2 \mu_{c} \frac{\partial^{2} w}{\partial x^{2}} \frac{\partial^{2} w}{\partial y^{2}}+\left(\frac{\partial^{2} w}{\partial y^{2}}\right)^{2} \\
& +2\left(1-\mu_{c}\right)\left(\frac{\partial^{2} w}{\partial x \partial y}\right)^{2}
\end{aligned}
$$

where $\operatorname{Re}($ ) and $\operatorname{Im}($ ) refer to the function of extracting real and imaginary part. It is worth noting that in (21) the maximum order of the polynomial expression of hardcoating materials is 3 . The practice has shown that for most conditions the 3-order polynomial can describe the material parameters with strain dependent characteristic accurately. In addition, it can be known from (18) to (22a), (22b), (22c), (22d), and (22e) that the material damping of both hard coating and metal substrate has included the above strain energy expression.

The kinemics energy $T$ of hard-coating composite plate can be described as

$$
T=\frac{1}{2}\left(\rho_{s} H_{s}+\rho_{c} H_{c}\right) \iint_{A}\left(\frac{\partial w}{\partial t}+\dot{u}_{g}\right)^{2} \mathrm{~d} A,
$$

where $\rho_{s}$ and $\rho_{c}$ are density of substrate and hard-coating material, respectively. Furthermore, one can define $m=$ $\rho_{s} H_{s}+\rho_{c} H_{c}$, which presents the mass of the unit area.

For the hard-coating composite plate structure, besides material damping of hard coating and substrate, the boundary conditions damping and fluid damping in air also should be contained and this damping can be equivalent to viscous damping, named as the remaining equivalent viscous damping in this work. The remaining equivalent viscous damping can also dissipate the energy of composite system and the expression is shown as follows:

$$
D=\frac{c}{2} \iint_{A}\left(\frac{\partial w}{\partial t}+\dot{u}_{g}\right)^{2} \mathrm{~d} A
$$

where $c$ is the coefficient of remaining equivalent viscous damping of composite plate.

Because only steady-state response of composite plate was considered in this study, Galerkin discrete method was adopted to reduce the order of the above nonlinear problem. In fact, in [13], similar method is also adopted to deal with the nonlinearity of hard-coating composite structure. It is assumed that the deflection expression of composite plate is

$$
w(x, y, t)=\sum_{j=1}^{N} \phi_{j}(x, y) \chi_{j}(t)
$$

where $\phi_{j}(x, y)$ is the $j$-order modal shape, which can be assumed consistent with the modal shape of uncoated plate, $\chi_{j}(t)$ is the $j$-order modal participation factor, and $N$ is the number of considered orders.

Substituting (25) into (19), the expression of linear strain energy can be changed as

$$
\begin{aligned}
U^{l} & =\frac{1}{2} \iint_{A}\left\{D _ { 1 } \left[\left(\sum_{j=1}^{N} \phi_{j}^{x x} \chi_{j}(t)\right)^{2}\right.\right. \\
& \left.+\left(\sum_{j=1}^{N} \phi_{j}^{y y} \chi_{j}(t)\right)^{2}\right]+2 D_{2}\left(\sum_{j=1}^{N} \phi_{j}^{x x} \chi_{j}(t)\right) \\
& \left.\cdot\left(\sum_{j=1}^{N} \phi_{j}^{y y} \chi_{j}(t)\right)+D_{3}\left(\sum_{j=1}^{N} \phi_{j}^{x y} \chi_{j}(t)\right)^{2}\right\} \mathrm{d} A
\end{aligned}
$$

where the meanings of operators of $\phi_{j}^{x x}, \phi_{j}^{y y}$, and $\phi_{j}^{x y}$ are $\partial^{2} \phi_{j}(x, y) / \partial x^{2}, \partial^{2} \phi_{j}(x, y) / \partial y^{2}$, and $\partial^{2} \phi_{j}(x, y) / \partial x \partial y$, respectively.

Moreover, to obtain the newer expression of the nonlinear strain energy, (25) is substituted into (21). It can be known from $(22 \mathrm{a}),(22 \mathrm{~b}),(22 \mathrm{c}),(22 \mathrm{~d})$, and $(22 \mathrm{e})$ that only $(22 \mathrm{~d})$ and $(22 \mathrm{e})$ need be transformed. Because $\phi_{j}(x, y)$ is real, the differential about it should be also real, so the imaginary part 
only exists in the $\chi_{j}(t)$, and then the expression of $f_{\text {eq }}$ and $g$ can be given as

$$
\begin{aligned}
f_{\mathrm{eq}} & =\sqrt{\left[\sum_{j=1}^{N} \phi_{j}^{x x} \operatorname{Re}\left(\chi_{j}\right)\right]^{2}+\left[\sum_{j=1}^{N} \phi_{j}^{x x} \operatorname{Im}\left(\chi_{j}\right)\right]^{2}} \\
& +\sqrt{\left[\sum_{j=1}^{N} \phi_{j}^{y y} \operatorname{Re}\left(\chi_{j}\right)\right]^{2}+\left[\sum_{j=1}^{N} \phi_{j}^{y y} \operatorname{Im}\left(\chi_{j}\right)\right]^{2}} \\
& +\sqrt{2\left(1-\mu_{c}\right)} \sqrt{\left[\sum_{j=1}^{N} \phi_{j}^{x y} \operatorname{Re}\left(\chi_{j}\right)\right]^{2}+\left[\sum_{j=1}^{N} \phi_{j}^{x y} \operatorname{Im}\left(\chi_{j}\right)\right]^{2}} \\
g & =\left(\sum_{j=1}^{N} \phi_{j}^{x x} \chi_{j}(t)\right)^{2} \\
& +2 \mu_{c}\left(\sum_{j=1}^{N} \phi_{j}^{x x} \chi_{j}(t)\right)^{2}\left(\sum_{j=1}^{N} \phi_{j}^{y y} \chi_{j}(t)\right)^{2} \\
& +\left(\sum_{j=1}^{N} \phi_{j}^{y y} \chi_{j}(t)\right)^{2}+2\left(1-\mu_{c}\right)\left(\sum_{j=1}^{N} \phi_{j}^{x y} \chi_{j}(t)\right)^{2} .
\end{aligned}
$$

Similarly, substituting (25) into (23) and (24), the newer expressions of kinemics energy and dissipation energy by the remaining equivalent viscous damping can be listed as follows:

$$
\begin{aligned}
& T=\frac{m}{2} \iint_{A}\left(\sum_{j=1}^{N} \phi_{j}(x, y) \dot{\chi}_{j}(t)+\dot{u}_{g}(t)\right)^{2} \mathrm{~d} A, \\
& D=\frac{c}{2} \iint_{A}\left(\sum_{j=1}^{N} \phi_{j}(x, y) \dot{\chi}_{j}(t)+\dot{u}_{g}(t)\right)^{2} \mathrm{~d} A .
\end{aligned}
$$

Arriving here, the expressions, such as strain energy $U$, kinemics energy $T$, and dissipation energy $D$ by the remaining equivalent viscous damping, are obtained by Galerkin discretion for the hard-coating plate structure. Substitute the above-mentioned expression into the following Lagrange equation:

$$
\begin{aligned}
\frac{\mathrm{d}}{\mathrm{d} t}\left(\frac{\partial T}{\partial \dot{\chi}_{l}(t)}\right)+\frac{\partial U}{\partial \chi_{l}(t)}+\frac{\partial D}{\partial \dot{\chi}_{l}(t)}=0, & \\
& (l=1, \ldots, N),
\end{aligned}
$$

and the final movement equation of hard-coating composite plate will be gotten.

Next, the derivatives of various energies to time $t, \chi_{l}(t)$ and $\dot{\chi}_{l}(t)$, are solved. It should be noted that the equivalent strain $\varepsilon_{e}$ is independent of time and the variable $f_{\text {eq }}$ in (26) does not include the time item yet, so they can be thought as constants during the differential calculation. Furthermore, if only the fundamental frequency vibration is considered, it can be assumed that $\chi_{j}(t)=\chi_{j} e^{i \omega t}$, which is introduced in the differential calculation. Then, by rearranging, the motion equation of the following style can be yielded:

$$
\left[\mathbf{K}_{\varepsilon}+i\left(\mathbf{C}_{1}+\omega \mathbf{C}_{2}\right)-\omega^{2} \mathbf{M}\right] \chi=\mathbf{F} .
$$

It can be noted that using this simple expression of solution the involved nonlinear problem has been transformed into solving the response vector $\chi$ independent of time. The other item in the above equation, $\mathbf{K}_{\varepsilon}$, is a nonlinear stiffness matrix considering the material nonlinearity of hard coating, $\mathbf{C}_{1}$ refers to the material damping matrix, $\mathbf{C}_{2}$ refers to the remaining equivalent viscous damping matrix, $\mathbf{M}$ is the mass matrix, and $\mathbf{F}$ is the excitation vector.

The matrixes $\mathbf{K}_{\varepsilon}, \mathbf{C}_{1}, \mathbf{C}_{2}$, and $\mathbf{M}$ are all $N$-order square matrix and the vectors $\boldsymbol{\chi}$ and $\mathrm{F}$ are $\mathrm{N}$-order vector. Additionally, $\mathbf{K}_{\varepsilon}+i \mathbf{C}_{1}$ can be considered as one item and expressed as complex matrix $\widetilde{\mathbf{K}}_{\varepsilon}^{*}$, and it is also an $N$-order square matrix.

The element of the $l$-row and $p$-column of complex matrix $\widetilde{\mathbf{K}}_{\varepsilon}^{*}$ can be shown as

$$
\begin{aligned}
& \widetilde{\mathbf{K}}_{\varepsilon}^{*}(l, p)=\iint_{A}\left[D_{1}\left(\phi_{l}^{x x} \phi_{p}^{x x}+\phi_{l}^{y y} \phi_{p}^{y y}\right)\right. \\
& \left.+D_{2}\left(\phi_{l}^{y y} \phi_{p}^{x x}+\phi_{p}^{y y} \phi_{l}^{x x}\right)+D_{3} \phi_{l}^{x y} \phi_{p}^{x y}\right] \mathrm{d} A \\
& \quad+\iint_{A}\left\{\left(D_{c 1}+D_{c 2} f_{\mathrm{eq}}+D_{c 3} f_{\mathrm{eq}}^{2}\right) f_{\mathrm{eq}} h\right\} \mathrm{d} A, \\
& h=\phi_{l}^{x x} \phi_{p}^{x x}+\phi_{l}^{y y} \phi_{p}^{y y}+\mu_{c}\left(\phi_{l}^{y y} \phi_{p}^{x x}+\phi_{p}^{y y} \phi_{l}^{x x}\right)+2(1 \\
& \left.\quad-\mu_{c}\right) \phi_{l}^{x y} \phi_{p}^{x y} .
\end{aligned}
$$

The element of the $l$-row and $p$-column of mass matrix $\mathbf{M}$ is

$$
\mathbf{M}(l, p)=m \iint_{A} \phi_{l} \phi_{p} \mathrm{~d} A .
$$

The element of the $l$-row and $p$-column of remaining equivalent viscous damping matrix $\mathbf{C}_{2}$ is

$$
\mathrm{C}_{2}(l, p)=c \iint_{A} \phi_{l} \phi_{p} \mathrm{~d} A .
$$

The damping coefficient $c$ can be simplified; for the $l$ order, the damping coefficient can be expressed by

$$
c_{l}=2 m \omega_{l} \zeta_{l}
$$

where $\omega_{l}$ is the $l$-order natural frequency and $\zeta_{l}$ is the $l$-order modal damping ratio which can be obtained by test.

For convenience, if ignoring the nondiagonal elements, the equivalent viscous damping matrix $\mathbf{C}_{2}$ can be further simplified. Then the matrix $\mathbf{C}_{2}$ became an $N$-order diagonal matrix and the element of the $l$-row and $l$-column is

$$
C_{2}(l, l)=2 m \omega_{l} \zeta_{l} \iint_{A} \phi_{l} \phi_{l} \mathrm{~d} A .
$$

Here, it was assumed that the boundary damping and air damping do not change before and after coating; then the modal damping ratio $\zeta_{l}$ can be introduced according to the damping of uncoated plate; similarly, $\omega_{l}$ refers to the natural frequency of uncoated plate.

The element of the excitation vector $\mathbf{F}$ can be described as

$$
\begin{aligned}
F(l)= & m \omega^{2} U_{G} \iint_{A} \phi_{l} \mathrm{~d} A-i \\
& \cdot 2 \omega \omega_{l} \zeta_{l} U_{G}\left(\rho_{s} H_{s}+\rho_{c} H_{c}\right) \iint_{A} \phi_{l} \mathrm{~d} A .
\end{aligned}
$$


In summary, the dynamics model of composite plate considering the strain material nonlinearity of hard coating was created. In this model, both the material damping and the remaining equivalent viscous damping are considered. Based on this model, the resonant frequencies of composite plate with the material nonlinearity and the nonlinear vibration response can be solved.

\section{Solution of Vibration Response and Resonant Frequency of Composite Plate}

4.1. Solution of Vibration Response. Due to the material nonlinearity of hard coating, (30) became a nonlinear algebraic equation. Here, the Newton-Raphson method was adopted to solve the vibration response of hard-coating composite plate. Equation (30) can be transformed as

$$
\mathbf{r}=\left[\mathbf{K}_{\varepsilon}+i\left(\mathbf{C}_{1}+\omega \mathbf{C}_{2}\right)-\omega^{2} \mathbf{M}\right] \boldsymbol{\chi}-\mathbf{F} .
$$

Because the residual value vector $\mathbf{r}$ contains the $\operatorname{Re}\left(\chi_{j}\right)$ and $\operatorname{Im}\left(\chi_{j}\right)$ items, also, each element of vector $\chi$ can be expressed as $\operatorname{Re}\left(\chi_{j}\right)+i \operatorname{Im}\left(\chi_{j}\right)$, so the Jacobi matrix $\mathbf{J}$ of vector $\mathbf{r}$ is needed and easily separated into the real part and imaginary part, which is shown as

$$
\mathbf{J}=\left[\begin{array}{cc}
\operatorname{Re}\left(\frac{\partial \mathbf{r}}{\partial \boldsymbol{\chi}_{R}}\right) & \operatorname{Re}\left(\frac{\partial \mathbf{r}}{\partial \boldsymbol{\chi}_{I}}\right) \\
\operatorname{Im}\left(\frac{\partial \mathbf{r}}{\partial \boldsymbol{\chi}_{R}}\right) & \operatorname{Im}\left(\frac{\partial \mathbf{r}}{\partial \boldsymbol{\chi}_{I}}\right)
\end{array}\right],
$$

where $\partial \mathbf{r} / \partial \chi_{R}$ is an $N$-order square matrix, and the element of $l$-row and $p$-column is

$$
\begin{aligned}
& \frac{\partial \mathbf{r}}{\partial \chi_{R}}(l, p)=\iint_{A}\left[D_{1}\left(\phi_{l}^{x x} \phi_{p}^{x x}+\phi_{l}^{y y} \phi_{p}^{y y}\right)\right. \\
& \left.\quad+D_{2}\left(\phi_{l}^{y y} \phi_{p}^{x x}+\phi_{p}^{y y} \phi_{l}^{x x}\right)+D_{3} \phi_{l}^{x y} \phi_{p}^{x y}\right] \mathrm{d} A \\
& \quad+i \omega c \iint_{A} \phi_{l} \phi_{p} \mathrm{~d} A-\omega^{2} m \iint_{A} \phi_{l} \phi_{p} \mathrm{~d} A+\left(D_{c 1}\right. \\
& \left.\quad+2 D_{c 2} f_{\mathrm{eq}}+3 D_{c 3} f_{\mathrm{eq}}^{2}\right) \psi \kappa+\left(D_{c 1}+D_{c 2} f_{\mathrm{eq}}\right. \\
& \left.\quad+D_{c 3} f_{\mathrm{eq}}^{2}\right) f_{\mathrm{eq}} h .
\end{aligned}
$$

Here,

$$
\begin{aligned}
\psi & =\frac{\sum_{j=1}^{N} \phi_{j}^{x x} \phi_{p}^{x x} \operatorname{Re}\left(\chi_{j}\right)}{\sqrt{\left[\sum_{j=1}^{N} \phi_{j}^{x x} \operatorname{Re}\left(\chi_{j}\right)\right]^{2}+\left[\sum_{j=1}^{N} \phi_{j}^{x x} \operatorname{Im}\left(\chi_{j}\right)\right]^{2}}} \\
& +\frac{\sum_{j=1}^{N} \phi_{j}^{y y} \phi_{p}^{y y} \operatorname{Re}\left(\chi_{j}\right)}{\sqrt{\left[\sum_{j=1}^{N} \phi_{j}^{y y} \operatorname{Re}\left(\chi_{j}\right)\right]^{2}+\left[\sum_{j=1}^{N} \phi_{j}^{y y} \operatorname{Im}\left(\chi_{j}\right)\right]^{2}}} \\
& +\sqrt{2\left(1-\mu_{c}\right)} \\
& \cdot \frac{\sum_{j=1}^{N} \phi_{j}^{x y} \phi_{p}^{x y} \operatorname{Re}\left(\chi_{j}\right)}{\sqrt{\left[\sum_{j=1}^{N} \phi_{j}^{x y} \operatorname{Re}\left(\chi_{j}\right)\right]^{2}+\left[\sum_{j=1}^{N} \phi_{j}^{x y} \operatorname{Im}\left(\chi_{j}\right)\right]^{2}}} \\
\kappa= & \sum_{j=1}^{N} \phi_{j}^{x x} \phi_{l}^{x x} \chi_{j}(t)+\sum_{j=1}^{N} \phi_{j}^{y y} \phi_{l}^{y y} \chi_{j}(t) \\
& +\mu_{c}\left[\sum_{j=1}^{N} \phi_{j}^{x x} \phi_{l}^{y y} \chi_{j}(t)+\sum_{j=1}^{N} \phi_{j}^{y y} \phi_{l}^{x x} \chi_{j}(t)\right] \\
& +2\left(1-\mu_{c}\right) \sum_{j=1}^{N} \phi_{j}^{x y} \phi_{l}^{x y} \chi_{j}(t) .
\end{aligned}
$$

Similarly, the element of $l$-row and $p$-column of matrix $\partial \mathbf{r} / \partial \boldsymbol{\chi}_{I}$ is

$$
\begin{aligned}
& \frac{\partial \mathbf{r}}{\partial \chi_{I}}(l, p)=\left(D_{c 1}+2 D_{c 2} f_{\mathrm{eq}}+3 D_{c 3} f_{\mathrm{eq}}^{2}\right) \Psi \kappa+i\left(D_{c 1}\right. \\
& \left.\quad+D_{c 2} f_{\mathrm{eq}}+D_{c 3} f_{\mathrm{eq}}^{2}\right) f_{\mathrm{eq}} h \\
& \quad+i\left\{\int \int _ { A } \left[D_{1}\left(\phi_{l}^{x x} \phi_{p}^{x x}+\phi_{l}^{y y} \phi_{p}^{y y}\right)\right.\right. \\
& \left.\quad+D_{2}\left(\phi_{l}^{y y} \phi_{p}^{x x}+\phi_{p}^{y y} \phi_{l}^{x x}\right)+D_{3} \phi_{l}^{x y} \phi_{p}^{x y}\right] \mathrm{d} A \\
& \left.\quad+i \omega c \iint_{A} \phi_{l} \phi_{p} \mathrm{~d} A-\omega^{2} m \iint_{A} \phi_{l} \phi_{p} \mathrm{~d} A\right\} .
\end{aligned}
$$

Here,

$$
\begin{aligned}
\Psi & =\frac{\sum_{j=1}^{N} \phi_{j}^{x x} \phi_{p}^{x x} \operatorname{Im}\left(\chi_{j}\right)}{\sqrt{\left[\sum_{j=1}^{N} \phi_{j}^{x x} \operatorname{Re}\left(\chi_{j}\right)\right]^{2}+\left[\sum_{j=1}^{N} \phi_{j}^{x x} \operatorname{Im}\left(\chi_{j}\right)\right]^{2}}} \\
& +\frac{\sum_{j=1}^{N} \phi_{j}^{y y} \phi_{p}^{y y} \operatorname{Im}\left(\chi_{j}\right)}{\sqrt{\left[\sum_{j=1}^{N} \phi_{j}^{y y} \operatorname{Re}\left(\chi_{j}\right)\right]^{2}+\left[\sum_{j=1}^{N} \phi_{j}^{y y} \operatorname{Im}\left(\chi_{j}\right)\right]^{2}}} \\
& +\sqrt{2\left(1-\mu_{c}\right)} \\
& \cdot \frac{\sum_{j=1}^{N} \phi_{j}^{x y} \phi_{p}^{x y}(x) \operatorname{Im}\left(\chi_{j}\right)}{\sqrt{\left[\sum_{j=1}^{N} \phi_{j}^{x y} \operatorname{Re}\left(\chi_{j}\right)\right]^{2}+\left[\sum_{j=1}^{N} \phi_{j}^{x y} \operatorname{Im}\left(\chi_{j}\right)\right]^{2}}}
\end{aligned}
$$


Extract the real and imaginary part of the vectors $\mathbf{r}$ and $\chi$, respectively, and form two new vectors, which are shown as follows:

$$
\begin{aligned}
\widehat{\mathbf{r}} & =\left[\begin{array}{ll}
\operatorname{Re}(\mathbf{r}) & \operatorname{Im}(\mathbf{r})
\end{array}\right]^{T}, \\
\widehat{\chi} & =\left[\begin{array}{ll}
\operatorname{Re}(\chi) & \operatorname{Im}(\chi)
\end{array}\right]^{T} .
\end{aligned}
$$

Then, the iterative formulas of Newton-Raphson method for (37) can be gotten:

$$
\begin{aligned}
\widehat{\mathbf{r}}^{(n)}+\mathbf{J}^{(n)} \cdot \Delta \hat{\boldsymbol{\chi}}^{(n)} & =0, \\
\hat{\boldsymbol{\chi}}^{(n+1)} & =\hat{\boldsymbol{\chi}}^{(n)}+\Delta \hat{\boldsymbol{\chi}}^{(n),}
\end{aligned}
$$

where $n$ is the iteration number.

Using the iterative calculation of (44a) and (44b), the solution with certain precision can be obtained and the precision of solution can be controlled by setting the maximum norm value of vector $\widehat{\mathbf{r}}$. When attaining termination conditions, the vector $\hat{\chi}$ should be transformed into the complex solution $\chi$, and then the deflection and strain of plate can be solved by (25) and (6). Finally, the vibration response of composite plate under base excitation is obtained by (17). The whole solution procedure is shown in Figure 3 and some steps are explained in the same figure.

For step 1, it is necessary to confirm the iterative initial value $\tilde{\chi}_{0}$, which can be obtained according to the following equation:

$$
\left[\mathbf{K}+i\left(\mathbf{C}_{1}+\omega \mathbf{C}_{2}\right)-\omega^{2} \mathbf{M}\right] \boldsymbol{\chi}=\mathbf{F},
$$

where the stiffness matrix $\mathbf{K}$ does not contain the strain dependent characteristic of hard-coating material; that is, the linear part of mechanical parameter of hard coating is introduced in the model according to $E_{c 0}^{*}=E_{\mathrm{cR} 0}+i E_{\mathrm{cI} 0}$. The element of the $l$-row and $p$-column of stiffness matrix $\mathbf{K}$ is

$$
\begin{aligned}
& K(l, p)=\iint_{A}\left[D_{1}\left(\phi_{l}^{x x} \phi_{p}^{x x}+\phi_{l}^{y y} \phi_{p}^{y y}\right)\right. \\
& \left.\quad+D_{2}\left(\phi_{l}^{y y} \phi_{p}^{x x}+\phi_{p}^{y y} \phi_{l}^{x x}\right)+D_{3} \phi_{l}^{x y} \phi_{p}^{x y}\right] \mathrm{d} A .
\end{aligned}
$$

For step 8 , the convergence condition is defined as norm 2 of vector $\mathbf{r}$ less than the set value, that is, $\|\mathbf{r}\|_{2} \leq$ TOL.

In the last step, the output values such as the deflection, strain, and displacement response of hard-coating composite plate are obtained by calculating the modules of the relative complex solution.

4.2. Solution of Resonant Frequency. When calculating resonant frequency of hard-coating composite plate, the resonant response of composite should be obtained in advance, so the calculation method of vibration response was described firstly at above section.

Neglecting the damping and excitation force items in (30), the characteristic equation considering the material nonlinearity of hard coating can be expressed as

$$
\left(\mathbf{K}_{\varepsilon}-\omega_{j}^{2} \mathbf{M}\right) \chi=0
$$

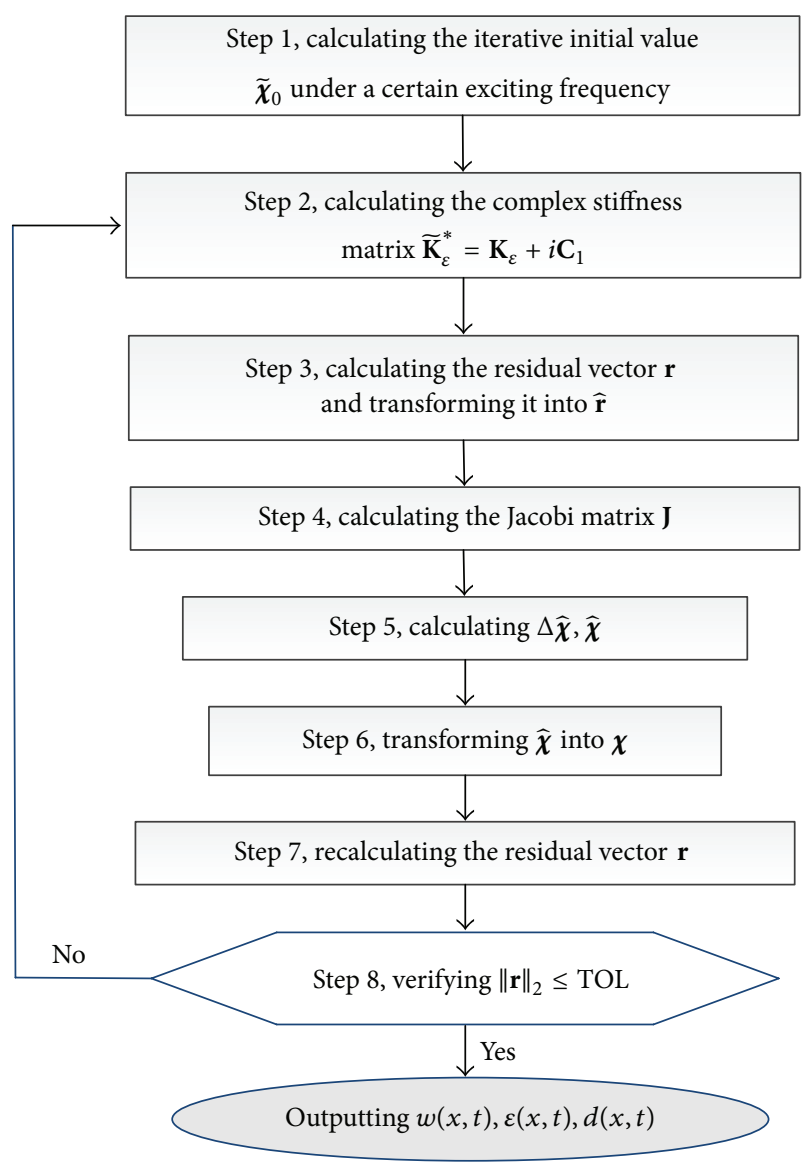

FIGURE 3: The procedure of solving vibration response of hardcoating composite plate.

Here, the iterative method is also adopted to solve the resonant frequency of composite plate. The calculating procedure is shown in Figure 4.

For step 1 , to obtain the initial value $\widetilde{\omega}_{j}$ of natural frequency, the material nonlinearity of hard coating is also not considered; the solution expression is

$$
\left(\mathbf{K}-\omega_{j}^{2} \mathbf{M}\right) \chi=0 .
$$

For step 2, corresponding to the initial value $\widetilde{\omega}_{j}$, the calculation equation of resonant response $\tilde{\chi}$ is

$$
\left[\mathbf{K}+i\left(\mathbf{C}_{1}+\omega_{j} \mathbf{C}_{2}\right)-\omega_{j}^{2} \mathbf{M}\right] \chi=\mathbf{F} .
$$

Similar to the response calculation, in step 6 , the convergence condition is defined as norm 2 of the difference between two adjacent frequencies; the expression is $\left\|\Delta \omega_{n}\right\|_{2} \leq$ TOL.

\section{Study Case and Discuss}

In the following, the titanium plate coated with $\mathrm{MgO}+\mathrm{Al}_{2} \mathrm{O}_{3}$ hard coating on one side was considered as a study case. The developed method was demonstrated to solve the dynamics characteristics considering the material nonlinearity of hard coating. 


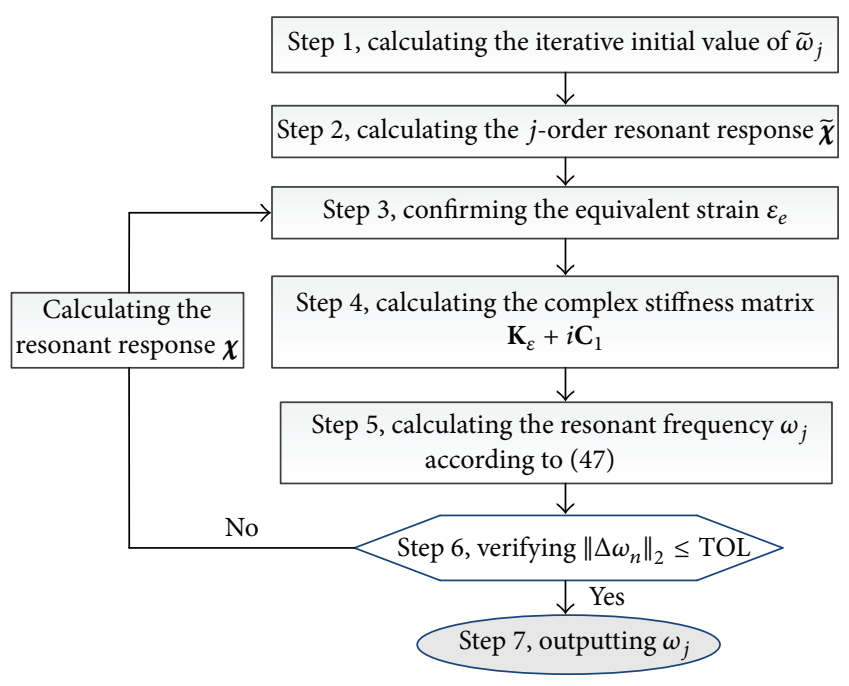

FIGURE 4: The procedure of solving resonant frequency of hardcoating composite plate.

5.1. Characterizing the Material Parameters of $\mathrm{MgO}+\mathrm{Al}_{2} \mathrm{O}_{3}$ Hard Coating by Polynomial. The material parameters of $\mathrm{MgO}+\mathrm{Al}_{2} \mathrm{O}_{3}$ hard coating are not constants and have strain dependent characteristic. In this study, the identification results of hard-coating material in [11] were introduced in the developed model. The storage modulus and loss modulus of $\mathrm{MgO}+\mathrm{Al}_{2} \mathrm{O}_{3}$ are shown in Figure 5.

It can be seen that these measured values cannot be fitted by linear function and have to be fitted by high order polynomial. Here, the cubic polynomial was chosen and the fitting curve is also shown in Figure 5.

The final expressions of material parameters of $\mathrm{MgO}+$ $\mathrm{Al}_{2} \mathrm{O}_{3}$ hard coating are

$$
\begin{aligned}
E_{\mathrm{cR}}\left(\varepsilon_{e}\right)= & 50.439-0.05386 \varepsilon_{e}+9.2396 \times 10^{-5} \varepsilon_{e}^{2} \\
& -5.4190 \times 10^{-8} \varepsilon_{e}^{3}, \\
E_{\mathrm{cI}}\left(\varepsilon_{e}\right)= & 0.9122+0.01321 \varepsilon_{e}-3.2997 \times 10^{-5} \varepsilon_{e}^{2} \\
& +2.2086 \times 10^{-8} \varepsilon_{e}^{3} .
\end{aligned}
$$

5.2. Vibration Experiment of Hard-Coating Composite Plate. The Ti-6Al-4V cantilever plate was chosen as study object and one side of plate was coated with $\mathrm{MgO}+\mathrm{Al}_{2} \mathrm{O}_{3}$ hard coating. The method of preparing hard coating was Air Plasma Spraying (APS) and the proportion of coating materials and depositing technology were similar to the ones mentioned in $[1,11]$. The geometry parameters of substrate and hard coating are listed in Table 1 and the densities of substrate and hard coating are $4420 \mathrm{~kg} / \mathrm{m}^{3}$ and $2565 \mathrm{~kg} / \mathrm{m}^{3}$, respectively. The material parameters of hard coating have the strain dependent characteristic, which are considered according to (50).

The experimental system is shown in Figure 6. In the system, the electromagnetic vibration shaker is base excitation equipment and the Doppler laser vibrometer is vibration
TABLE 1: The geometry parameters of hard-coating composite plate.

\begin{tabular}{lcccc}
\hline & Material type & $\begin{array}{c}\text { Length/ } \\
\mathrm{mm}\end{array}$ & $\begin{array}{c}\text { Width/ } \\
\mathrm{mm}\end{array}$ & $\begin{array}{c}\text { Thickness/ } \\
\mathrm{mm}\end{array}$ \\
\hline Substrate & $\mathrm{Ti}-6 \mathrm{Al}-4 \mathrm{~V}$ & 134 & 110 & 1.0 \\
Hard coating & $\mathrm{MgO}+\mathrm{Al}_{2} \mathrm{O}_{3}$ & 134 & 110 & 0.02 \\
\hline
\end{tabular}

TABle 2: The instruments used in this test.

\begin{tabular}{lc}
\hline Number & Name \\
\hline 1 & LMS SCADAS mobile front-end \\
2 & KINGDESIGN EM-1000F shaker \\
3 & Polytec PDV-100 laser vibrometer \\
4 & BK 4514-001 acceleration sensor \\
5 & High-performance notebook computers \\
\hline
\end{tabular}

picking equipment. The vibration picking point locates at the lower part of composite plate, and the coordinate values are $x=26.8 \mathrm{~mm}, y=22 \mathrm{~mm}$, according to the coordinates shown in Figure 6. Table 2 lists the instruments used in this test in detail. It should be noted that an acceleration sensor was placed in the fixture and this sensor was used to monitor the base excitation amplitude of fixture. Another acceleration sensor was used to control the amplitude of vibration shaker. It has been found that the measurement values are different between the two accelerators. In this study, the measurement value in the fixture was chosen as the base excitation amplitude. To ensure having consistent boundary conditions for the uncoated and coated plate, the torque wrench was chosen and the tightening torque of bolt is $34 \mathrm{~N} \cdot \mathrm{m}$ in the fixture.

Herein, the 6-order resonant frequency and resonant response calculation of hard-coating composite plate were chosen to display the feasibility of the proposed method. The reason of choosing the 6-order vibration characteristic was that the effect of boundary condition on the dynamics characteristics of higher order of composite plate is small. According to the needs of analytical calculation and verifying the analytical calculation results, the first 8-order modal damping ratios of uncoated plate, the natural frequencies of composite plate, and the 6-order resonant frequency and resonant response under different excitation levels $(0.1 \mathrm{~g}$, $0.3 \mathrm{~g}, 0.4 \mathrm{~g}, 0.5 \mathrm{~g}, 0.6 \mathrm{~g}$, and $0.7 \mathrm{~g}$ ) are tested. Among them, the modal damping ratios of uncoated plate were obtained by half-power bandwidth method from the sweeping response of composite plate under $0.1 \mathrm{~g}$. The natural frequencies of composite plate were gotten from the $3 \mathrm{D}$ waterfall figure of sweeping response under $0.1 \mathrm{~g}$. In this study, $0.1 \mathrm{~g}$ excitation level was thought to be very low, which can not lead to the appearance of material nonlinearity of hard coating, so the natural frequencies obtained by experiment were thought as linear natural frequencies. The 6-order resonant frequency and resonant response were also acquired by the $3 \mathrm{D}$ waterfall figure of sweeping response and the sweeping direction was from high frequency to low frequency. All the tested results are listed in Table 3 to Table 5. 


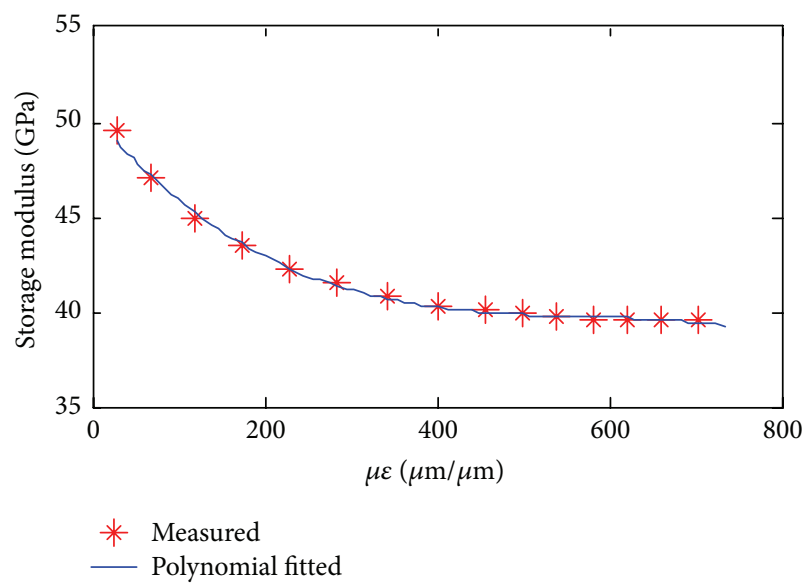

(a) Storage modulus

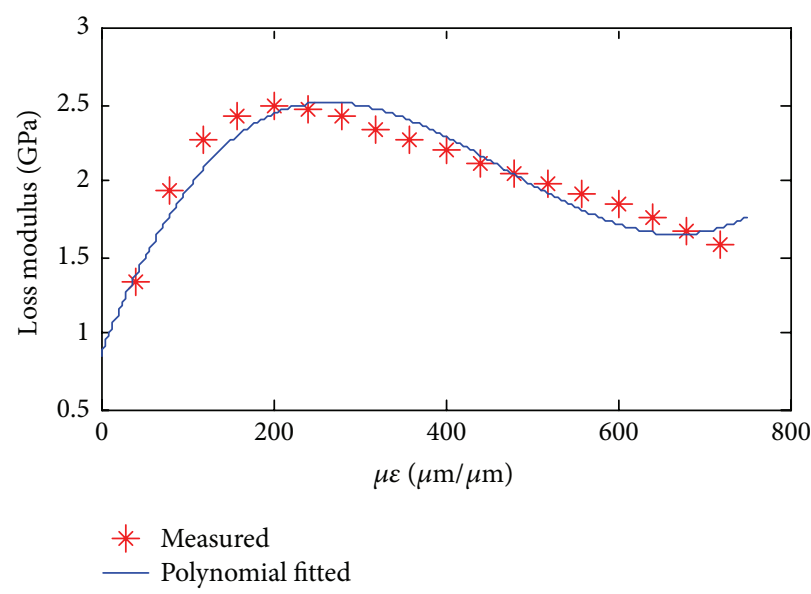

(b) Loss modulus

FIGURE 5: Mechanical parameters of $\mathrm{MgO}+\mathrm{Al}_{2} \mathrm{O}_{3}$ with strain dependent characteristics.

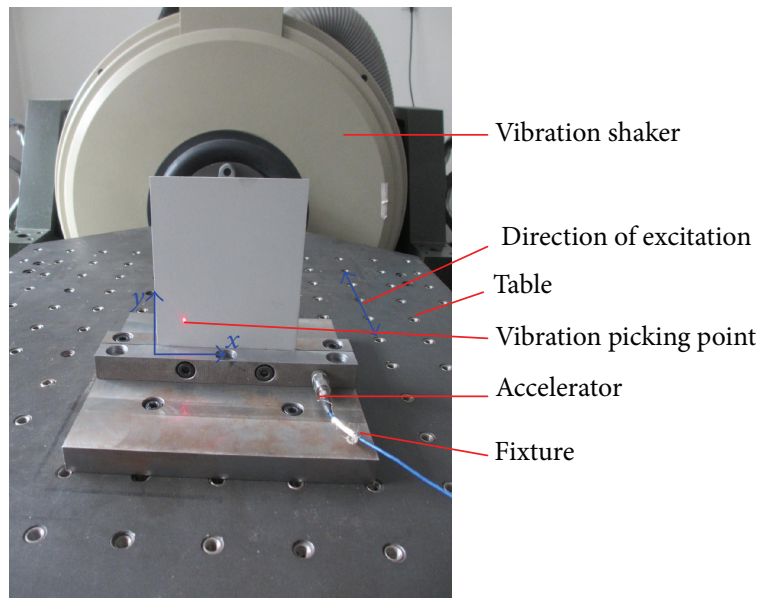

FIGURE 6: Experimental system for hard-coating cantilever plate.

It can be seen from Table 5 that the 6 -order resonant frequency of composite plate decreases with the increase of excitation levels. The sweeping response in the considered frequency range is shown in Figure 7. It should be explained that the labels of excitation levels in Figure 7 were the setting amplitude of vibration table, which are used to control the vibration shaker. Also, it can be seen from Table 5 that the excitation level in the fixture was less than the excitation level in the table. Maybe, the reason is that the energy is dissipated when transferring from table to fixture.

5.3. Analytical Calculation. The strain dependent characteristic of hard coating is considered for the proposed method, so the solving process using the proposed method is named as nonlinear calculation. In contrast, if not considering the strain dependent characteristic, the solving process is named as linear calculation. To compare with the proposed method, the linear calculation was also done for the hard-coating

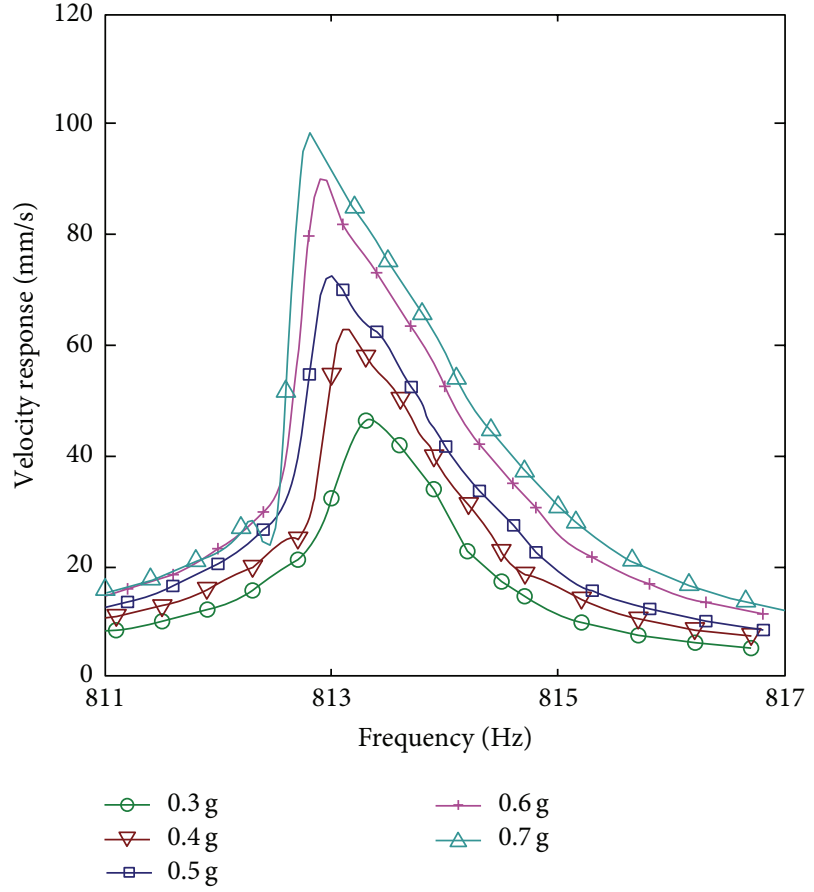

FIgURE 7: The 6-order sweeping response of composite plate under different excitation levels.

composite plate. For the linear calculation, the material parameters of hard coating were taken as follows: storage modulus $50.439 \mathrm{GPa}$ and loss modulus $0.918 \mathrm{GPa}$, which are corresponding to the 0 strains in Figure 5. The picking vibration point was consistent with experiment. Using these parameters, the resonant frequencies and the 6-order resonant response under different excitation levels were solved. For the nonlinear calculation, the convergence conditions of both vibration response and resonant frequency calculation were defined as TOL $=0.001$. The relative results are listed or 
TABLE 3: The first 8-order modal damping ratios of uncoated plate obtained by experiment.

\begin{tabular}{lcccccccc}
\hline Order & 1 & 2 & 3 & 4 & 5 & 6 & 7 & 8 \\
\hline Damping ratio/\% & 0.7953 & 0.1937 & 0.03542 & 0.03413 & 0.04231 & 0.06831 & 0.05253 & 0.04324 \\
\hline
\end{tabular}

TABLE 4: The first 8-order natural frequencies of composite plate obtained by experiment and linear calculation/Hz.

\begin{tabular}{lcccccccc}
\hline Order & 1 & 2 & 3 & 4 & 5 & 6 & 7 & 8 \\
\hline Experiment $f^{t}$ & 47.0 & 132.10 & 287.5 & 460.4 & 509.1 & 813.4 & 909.3 & 989.2 \\
Linear calculation $f^{l}$ & 46.7 & 132.6 & 289.4 & 463.4 & 505.4 & 820.9 & 901.1 & 995.3 \\
Difference $\left|f^{l}-f^{t}\right| / f^{t}$ & $0.64 \%$ & $0.37 \%$ & $0.66 \%$ & $0.65 \%$ & $0.72 \%$ & $0.92 \%$ & $0.90 \%$ & $0.62 \%$ \\
\hline
\end{tabular}

TABLE 5: The 6-order resonant frequency and resonant response under different excitation levels obtained by experiment.

\begin{tabular}{lccccc}
\hline Excitation level in the table/g & 0.3 & 0.4 & 0.5 & 0.6 & 0.7 \\
Excitation level in the fixture/g & 0.23 & 0.30 & 0.38 & 0.47 & 0.54 \\
Resonant frequency/Hz & 813.35 & 813.15 & 813.00 & 812.90 & 812.80 \\
Resonant response/(mm/s) & 46.684 & 63.366 & 72.571 & 90.105 & 98.518 \\
\hline
\end{tabular}

TABLE 6: The 6-order resonant frequency and resonant response under different excitation levels obtained by linear and nonlinear calculation.

\begin{tabular}{lccc}
\hline Excitation levels/g & Calculation type & Resonant frequency/Hz & Resonant response/(mm/s) \\
\hline \multirow{2}{*}{0.3} & Linear & 820.89 & 50.183 \\
& Nonlinear & 820.64 & 47.574 \\
\hline \multirow{2}{*}{0.4} & Linear & 820.89 & 65.457 \\
& Nonlinear & 820.58 & 61.171 \\
\hline \multirow{2}{*}{0.5} & Linear & 820.89 & 82.912 \\
& Nonlinear & 820.50 & 76.319 \\
\hline \multirow{2}{*}{0.7} & Linear & 820.89 & 102.549 \\
& Nonlinear & 820.42 & 92.928 \\
\hline & Linear & 820.89 & 117.822 \\
\end{tabular}

shown in Tables 4 and 6 and Figure 8, and so forth. It can be noted from Table 4 that the maximum difference of the first 8 order natural frequencies between the experiment and linear calculation is less than $1 \%$, and the discussion about the other results can be seen in the following section.

5.4. Results Comparison and Difference Analysis. In this section, the linear and nonlinear calculation results for the 6-order resonant frequency and resonant response were compared with the experimental values. The comparison of resonant response is listed in Table 7 and the comparison of resonant frequency is listed in Table 8 .

From Table 7, a general rule can be still drawn; that is, the difference between linear calculation of resonant response and experiment values becomes bigger with the increase of excitation levels, although there are exception conditions when the excitation levels are $0.3 \mathrm{~g}$ and $0.6 \mathrm{~g}$. For example, when the excitation level is less than $0.4 \mathrm{~g}$, the maximum difference is $7.49 \%$, and when the excitation level is bigger than this value, the difference increases obviously and the maximum value has achieved $19.59 \%$ during this calculation. Compared with the linear calculation, the nonlinear calculation proposed in this study can always maintain an acceptable precision and the maximum difference with experiment value is less than $7.5 \%$. For the resonant frequency calculation listed in Table 8, similar conclusion can be drawn; that is, when the excitation level is bigger, the nonlinear calculation should be adopted, although for this case the precisions of both the linear and nonlinear calculation are acceptable. By the above comparison, the rationality of the calculation method considering the material nonlinearity of hard coating was verified.

\section{Conclusions}

On the basis of considering the material nonlinearity of hard coating, the dynamics modeling and analysis for a hardcoating composite plate were studied, and some important conclusions are listed as follows. 


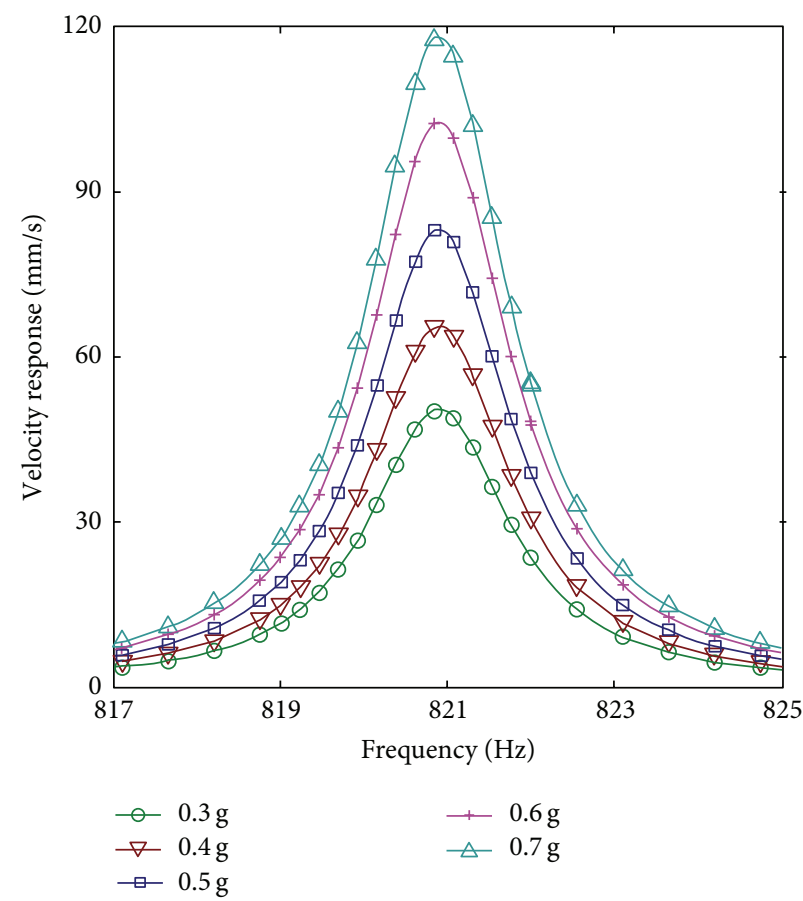

(a) Linear calculation

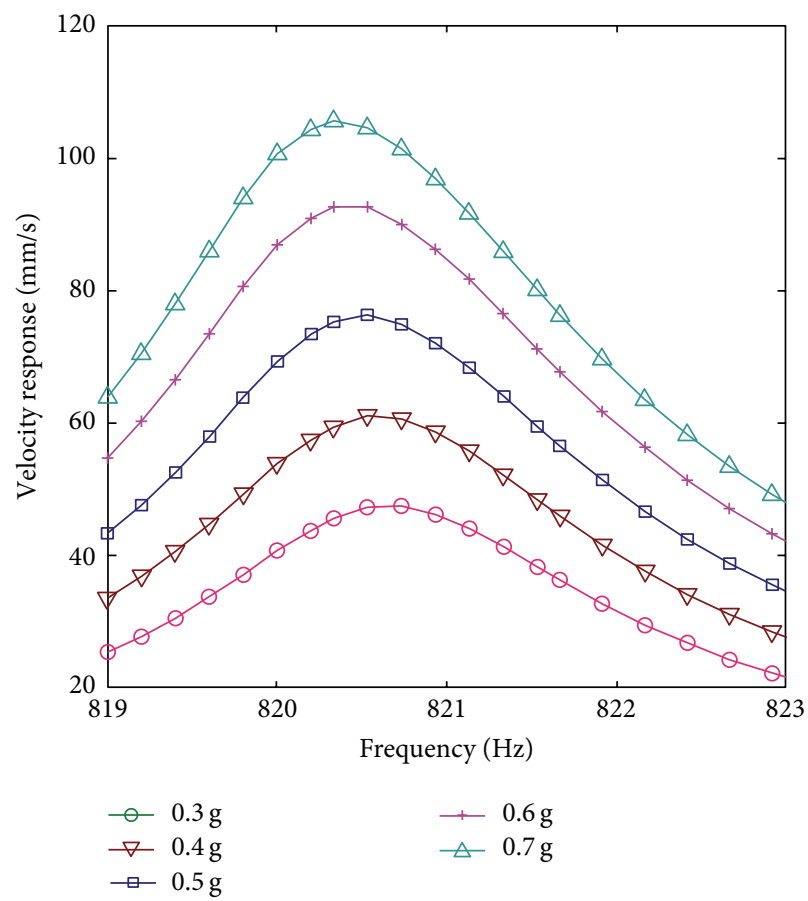

(b) Nonlinear calculation

FIGURE 8: Frequency domain response of hard-coating composite plate near the 6-order resonance region.

TABLE 7: The comparison of resonant response $/ \mathrm{mm} / \mathrm{s}$.

\begin{tabular}{lccccc}
\hline \multirow{2}{*}{ Item } & \multicolumn{5}{c}{ Excitation level/g } \\
& 0.3 & 0.4 & 0.5 & 0.6 & 0.7 \\
\hline Experiment $A^{t}$ & 46.684 & 63.366 & 72.571 & 90.105 & 98.518 \\
\hline $\begin{array}{l}\text { Linear calculation } \\
A^{l}\end{array}$ & 50.183 & 65.457 & 82.912 & 102.549 & 117.822 \\
\hline $\begin{array}{l}\text { Difference } \\
\left|A^{l}-A^{t}\right| / A^{t}\end{array}$ & $7.49 \%$ & $3.3 \%$ & $14.25 \%$ & $13.81 \%$ & $19.59 \%$ \\
\hline $\begin{array}{l}\text { Nonlinear } \\
\text { calculation } A^{\mathrm{nl}}\end{array}$ & 47.574 & 61.171 & 76.319 & 92.928 & 105.570 \\
$\begin{array}{l}\text { Difference } \\
\left|A^{\mathrm{nl}}-A^{t}\right| / A^{t}\end{array}$ & $1.90 \%$ & $3.46 \%$ & $5.16 \%$ & $3.13 \%$ & $7.15 \%$ \\
\hline
\end{tabular}

(1) Using the high order polynomial to characterize the material parameters of hard coating is an effective method. On the one hand, the high order polynomial can describe the change of mechanical parameter with the strain amplitude; on the other hand, the mechanical parameters expressed by polynomial can be easily introduced into the analytical model.

(2) The created analytical model and solving method in this study can correctly solve the dynamics characteristics. The practice for the 6-order calculation of hardcoating composite plate shows that the maximum difference of the resonant response calculation is less than $7.5 \%$ and the maximum difference of the resonant frequency calculation is less than $0.93 \%$.
TABLE 8: The comparison of resonant frequency/Hz.

\begin{tabular}{lccccc}
\hline \multirow{2}{*}{ Item } & \multicolumn{5}{c}{ Excitation level/g } \\
& 0.3 & 0.4 & 0.5 & 0.6 & 0.7 \\
\hline Experiment $f^{t}$ & 813.35 & 813.15 & 813.00 & 812.90 & 812.80 \\
\hline $\begin{array}{l}\text { Linear calculation } \\
f^{l}\end{array}$ & 820.89 & 820.89 & 820.89 & 820.89 & 820.89 \\
\hline $\begin{array}{l}\text { Difference } \\
\left|f^{l}-f^{t}\right| / f^{t}\end{array}$ & $0.93 \%$ & $0.95 \%$ & $0.97 \%$ & $0.98 \%$ & $0.99 \%$ \\
\hline $\begin{array}{l}\text { Nonlinear } \\
\text { calculation } f^{\mathrm{nl}}\end{array}$ & 820.64 & 820.58 & 820.50 & 820.42 & 820.37 \\
\hline $\begin{array}{l}\text { Difference } \\
\left|f^{\mathrm{nl}}-f^{t}\right| / f^{t}\end{array}$ & $0.89 \%$ & $0.91 \%$ & $0.92 \%$ & $0.92 \%$ & $0.93 \%$ \\
\hline
\end{tabular}

(3) The resonant response values between linear and nonlinear calculation are significantly different and the difference between linear calculation and experiment values becomes bigger with the increase of excitation levels. So, when the excitation level is bigger, the material nonlinearity of hard coating must be considered; using the nonlinear calculation is needed to acquire the dynamics characteristics of hard-coating composite structure.

(4) By comparison of the resonant responses obtained by linear and nonlinear calculation for the hard-coating composite plate, it can be found that the resonant response obtained by nonlinear calculation is less than the result of linear calculation, which maybe 
means that the material nonlinearity of hard coating is good to the vibration reduction effect.

\section{Conflict of Interests}

The authors declare that there is no conflict of interests regarding the publication of this paper.

\section{Acknowledgments}

This project was supported by National Natural Science Foundation of China (Grant no. 51375079) and the Fundamental Research Funds for the Central Universities of China (Grant no. N140301001). In addition, the first author (Wei Sun) would like to thank the University of Akron for providing a Visiting Professor position to carry out this research work.

\section{References}

[1] C. Blackwell, A. Palazotto, T. J. George, and C. J. Cross, "The evaluation of the damping characteristics of a hard coating on titanium," Shock and Vibration, vol. 14, no. 1, pp. 37-51, 2007.

[2] F. Ivancic and A. Palazotto, "Experimental considerations for determining the damping coefficients of hard coatings," Journal of Aerospace Engineering, vol. 18, no. 1, pp. 8-17, 2005.

[3] N. Tassini, S. Patsias, and K. Lambrinou, "Ceramic coatings: a phenomenological modeling for damping behavior related to microstructural features," Materials Science and Engineering A, vol. 442, no. 1-2, pp. 509-513, 2006.

[4] P. J. Torvik, "A slip damping model for plasma sprayed ceramics," Journal of Applied Mechanics, vol. 76, no. 6, Article ID 061018, 2009.

[5] R. K. A. Al-Rub and A. N. Palazotto, "Micromechanical theoretical and computational modeling of energy dissipation due to nonlinear vibration of hard ceramic coatings with microstructural recursive faults," International Journal of Solids and Structures, vol. 47, no. 16, pp. 2131-2142, 2010.

[6] J. Green and S. Patsias, "A preliminary approach for the modeling of a hard damping coating using friction elements," in Proceedings of the 7th National Turbine Engine High Cycle Fatigue Conference, pp. 7c1-7c9, Palm Beach, Fla, USA, May 2002.

[7] S. A. Reed, Development of experimental, analytical, and numerical approximations appropriate for nonlinear damping coatings [Ph.D. thesis], Air Force Institute of Technology, Greene County, Ohio, USA, 2007.

[8] P. J. Torvik and J. Hansel, "Mechanical properties of a ceramic coating with vem infiltration," Journal of Engineering Materials and Technology, vol. 131, no. 3, Article ID 031003, 2009.

[9] P. J. Torvik, "Determination of mechanical properties of nonlinear coatings from measurements with coated beams," International Journal of Solids and Structures, vol. 46, no. 5, pp. 10661077, 2009.

[10] S. Patsias, C. Saxton, and M. Shipton, "Hard damping coatings: an experimental procedure for extraction of damping characteristics and modulus of elasticity," Materials Science and Engineering A, vol. 370, no. 1-2, pp. 412-416, 2004.

[11] S. A. Reed, A. N. Palazotto, and W. P. Baker, "An experimental technique for the evaluation of strain dependent material properties of hard coatings," Shock and Vibration, vol. 15, no. 6, pp. 697-712, 2008.

[12] N. Tassini, K. Lambrinou, I. Mircea, M. Bartsch, S. Patsias, and O. Van der Biest, "Study of the amplitude-dependent mechanical behaviour of yttria-stabilised zirconia thermal barrier coatings," Journal of the European Ceramic Society, vol. 27, no. 2-3, pp. 1487-1491, 2007.

[13] S. Filippi and P. J. Torvik, "A methodology for predicting the response of blades with nonlinear coatings," Journal of Engineering for Gas Turbines and Power, vol. 133, no. 4, Article ID 042503, 2011.

[14] G. Parthasarathy, C. V. R. Reddy, and N. Ganesan, "Partial coverage of rectangular plates by unconstrained layer damping treatments," Journal of Sound and Vibration, vol. 102, no. 2, pp. 203-216, 1985. 


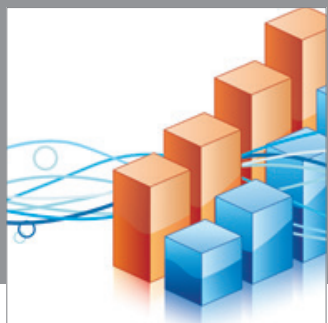

Advances in

Operations Research

mansans

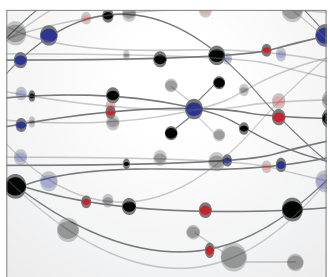

The Scientific World Journal
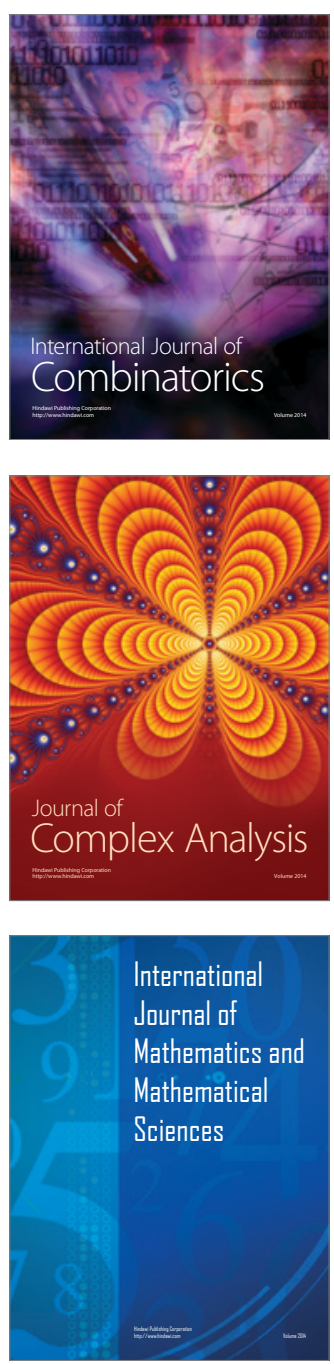
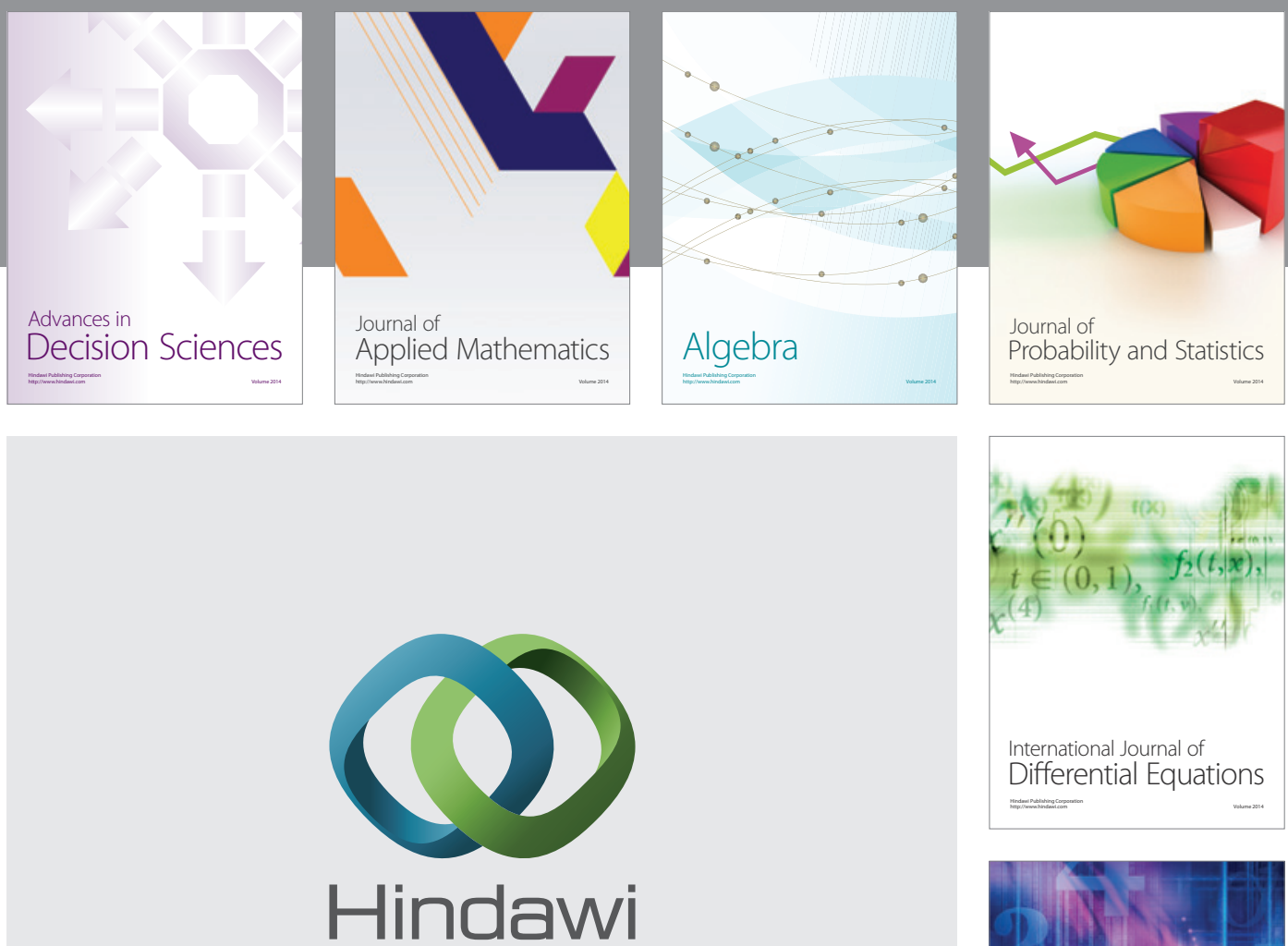

Submit your manuscripts at http://www.hindawi.com
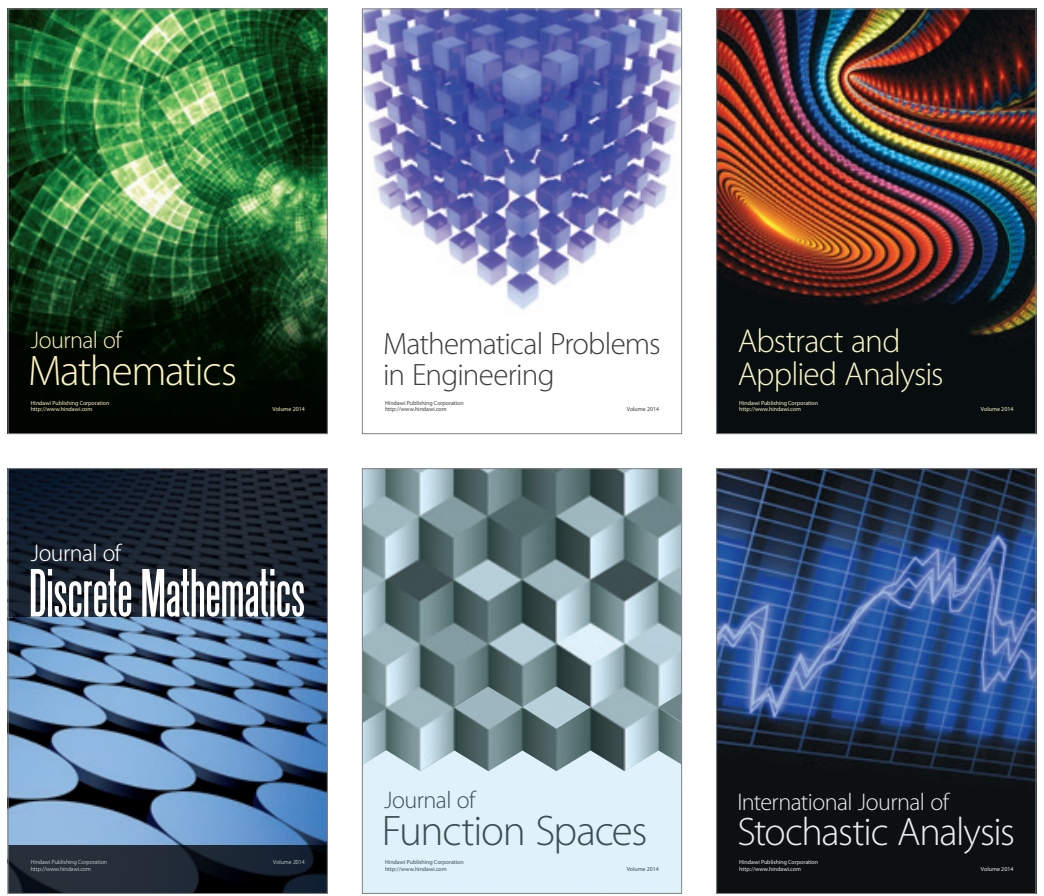

Journal of

Function Spaces

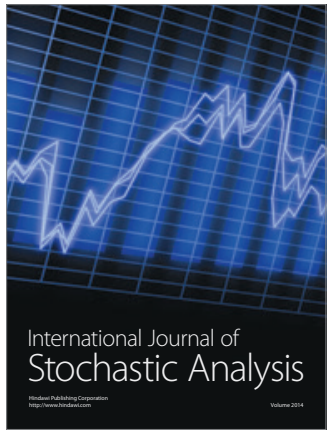

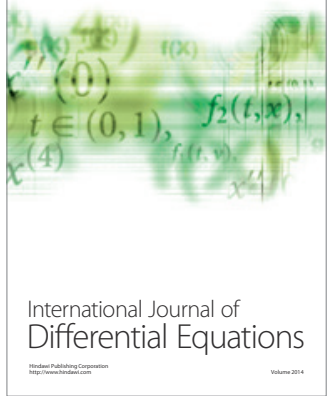
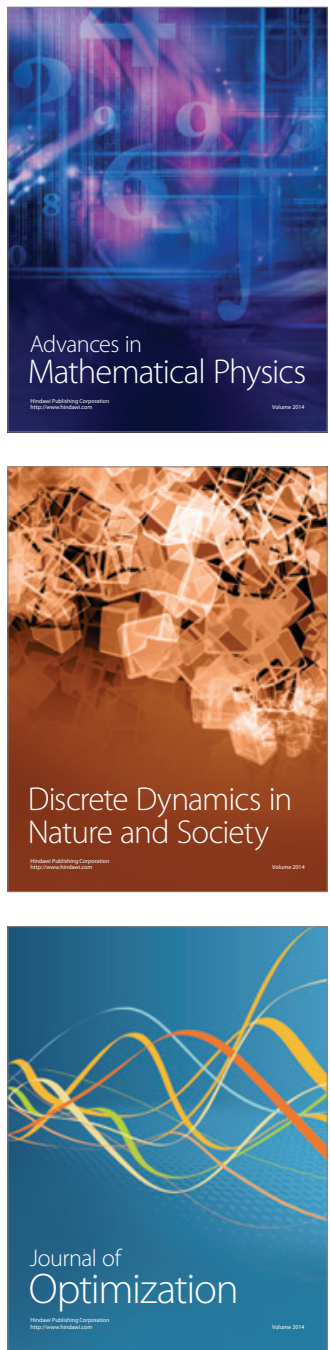